\title{
Measurements of Fission Products Yields with the LOHENGRIN mass spectrometer at ILL
}

\author{
A. Chebboubi ${ }^{1, a}$, S. Julien-Laferrière ${ }^{1}$, J. Nicholson ${ }^{1}$, G. Kessedjian ${ }^{2}$, O. Serot ${ }^{1}$, A. Blanc ${ }^{3}$, D. \\ Bernard $^{1}$, H. Faust ${ }^{3}$, Y.H. Kim ${ }^{3}$, U. Köster ${ }^{3}$, A. Letourneau ${ }^{4}$, O. Litaize ${ }^{1}$, O. Méplan², P. Mutti ${ }^{3}$, M. \\ Rapala $^{4}$, M. Ramdhane ${ }^{2}$, and C. Sage ${ }^{2}$ \\ ${ }^{1}$ CEA, DEN, DER, SPRC, Cadarache, Physics Studies Laboratory, F-13108 Saint-Paul-lès-Durance, France \\ ${ }^{2}$ LPSC, Université Grenoble-Alpes, CNRS/IN2P3, F-38026 Grenoble Cedex, France \\ ${ }^{3}$ Institut Laue-Langevin, F-38042 Grenoble Cedex 9, France \\ ${ }^{4}$ IRFU, CEA,Université Paris-Saclay, 91191 Gif-sur-Yvette, France
}

\begin{abstract}
The CEA in collaboration with ILL and LPSC has developed a measurement program on symmetric and heavy mass fission product distributions. The combination of measurements with ionisation chamber and Ge detectors is necessary to describe precisely the heavy fission product region in mass and charge. Recently, new measurements of fission yields and kinetic energy distributions, for different fissioning systems $\left({ }^{233,235} \mathrm{U}\left(n_{t h}, f\right),{ }^{241} \mathrm{Am}\left(2 n_{t h}, f\right)\right.$ and $\left.{ }^{239,241} \mathrm{Pu}\left(n_{t h}, f\right)\right)$, were performed with recoil spectrometer LOHENGRIN. The focus has been done on the self-normalization of the data to provide new absolute measurements, independently of any libraries along with the experimental covariance matrix. To reach precise measurements, a new experimental procedure was developed along with a new analysis method.
\end{abstract}

\section{Introduction}

The mass and isotopic yields of the fission products have a direct influence on the amount of neutron poisons that limit the fuel burnup and on the evaluation of the residual power of the reactor after shutdown $[1,2]$. The fission yields of the plutonium nuclides are also mandatory for the studies on the fuel multi-recycling [3]. In order to significantly improve the precision of nuclear data, more and more fundamental fission models are used in the evaluation processing. Therefore, tests of fission models become a central issue to achieve coherent libraries of nuclear data. In this framework, an important investigation in the experimental limits of facilities is required to provide complete sets of data with their coherent variance-covariance matrices. In the past with the LOHENGRIN spectrometer [4] of the Institut Laue-Langevin (ILL), priority has been given for the studies in the light fission fragment mass range. This approach was due to the opportunity to extract the mass and charge distribution using a double Frisch grid ionization chamber (IC). Since 2010, the CEA in collaboration with ILL and the LPSC study the fission products properties by combining IC measurements with $\gamma$ spectroscopy using High Purity Germanium (HPGe) detectors. These studies are focused on the heavy mass region. Along he years, several fissioning systems such as ${ }^{239} \mathrm{Pu}\left(n_{t h}, f\right)[5,6],{ }^{241} \mathrm{Am}\left(2 n_{t h}, f\right)$ [7],

\footnotetext{
ae-mail: abdelhazize.chebboubi@cea.fr
} 
${ }^{235} \mathrm{U}\left(n_{t h}, f\right)[8],{ }^{233} \mathrm{U}\left(n_{t h}, f\right)[9]$ and ${ }^{241} \mathrm{Pu}\left(n_{t h}, f\right)$ [10] were measured. In this article, a focus on the mass yield measurements is made. Then some results on isotopic yields and complementary observables are shown.

\section{Measurements of fission product mass yields $Y(A)$}

The measurement of $Y(A)$ were done at the LOHENGRIN recoil separator of the ILL. The different parts of the LOHENGRIN spectrometer are shown on Fig. 1 (left). The fissile isotope target is placed near the core of the reactor under a thermal neutron flux about $5 \times 10^{14} \mathrm{n} \cdot \mathrm{cm}^{-2} \cdot \mathrm{s}^{-1}$. The combination of a magnetic and electric fields allows a separation of the produced fission products according to their ratios mass $A$ over ionic charge $q$ and kinetic energy $E_{k}$ over ionic charge. Finally a refocusing magnet [11] permits to increase the particle density at the focal position 2. To remove the mass degeneracy an ionization chamber (at the focal position 1) is used to measure $E_{k}$. Figure 1 (right) shows the energy loss inside the first part of the IC as function of the total energy loss inside the IC. Each spot is then associated to a mass number.
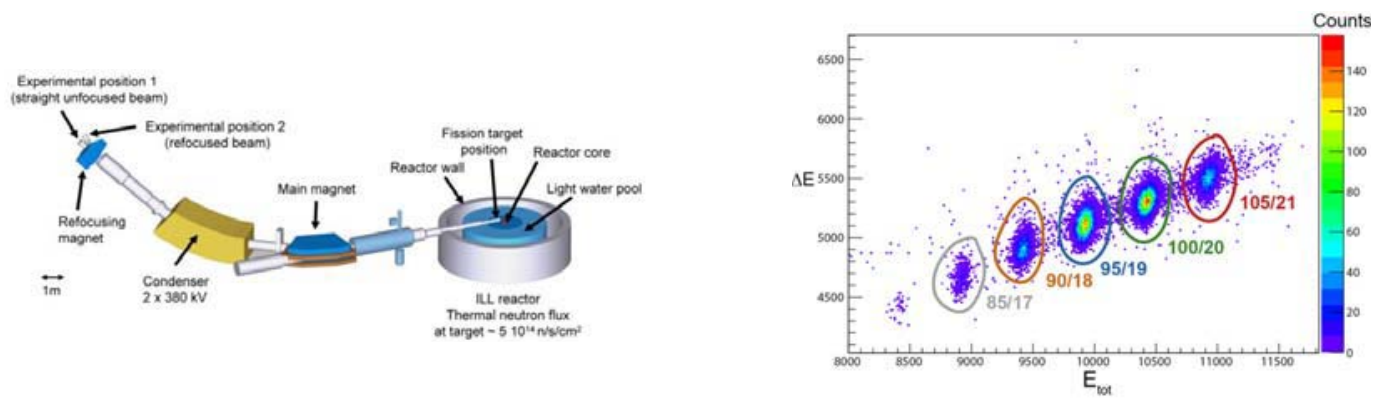

Figure 1. Scheme of the LOHENGRIN spectrometer (left). Plot of the energy loss $\Delta E$ in the first part of the IC and the total energy loss $E_{\text {tot }}$ inside the IC. Each spot corresponds to a kinetic energy, which refers to the fission product mass (right). The mentionned numbers correspond to the ratio A/q.

By definition, the relative mass yield $\mathcal{N}(A)$ is written :

$$
\mathcal{N}(A)=\sum_{q} \int_{E_{k}} \mathcal{N}\left(A, q, E_{k}\right) d E_{k}
$$

with $\mathcal{N}\left(A, q, E_{k}\right)$ the count rate for a given ionic charge and kinetic energy selected by the LOHENGRIN spectrometer. However because of the finite energy resolution of the spectrometer, measurement by energy bins must be done. Also because of the high flux, the fissile matter evolves quite rapidly with time, typically 20 days of irradiation for a ${ }^{241} \mathrm{Pu}$ sample of $100 \mu \mathrm{g} . \mathrm{cm}^{-2}$. In order to measure the maximum number of masses (during a single experimental campaign), some approximations are then made:

$$
\mathcal{N}\left(A, q, E_{k}\right)=\frac{N_{i o n}\left(A, q, E_{k} \pm \Delta E_{k}, t\right)}{B U(t) \times \Delta E_{k}}
$$

with $N_{i o n}\left(A, q, E_{k} \pm \Delta E_{k}, t\right)$ the count rate extracted from the IC at a given kinetic energy, ionic charge and time. The kinetic energy resolution depends on the aperture at the exit of the condenser. Here the kinetic energy resolution is: $\Delta E_{k}=0.005 \times E_{k}$, which corresponds to an aperture of $3.24 \mathrm{~cm}^{1}$. If

\footnotetext{
${ }^{1}$ In the following, $E_{k}$ refers to $E_{k} \pm \Delta E_{k}$
} 
we suppose that the ionic charge and the kinetic energy are two independent parameters, then eq. (1) becomes:

$$
\begin{aligned}
\mathcal{N}(A) & =\frac{\sum_{E_{k}} \mathcal{N}\left(A, q_{m}, E_{k}\right)}{P\left(q_{m}, E_{k}^{m}\right)} \\
P\left(q_{m}, E_{k}^{m}\right) & =\frac{\mathcal{N}\left(A, q_{m}, E_{k}^{m}\right)}{\sum_{q} \mathcal{N}\left(A, q, E_{k}^{m}\right)}
\end{aligned}
$$

with $P\left(q_{m}, E_{k}^{m}\right)$ the probability to measure the ionic charge $q_{m}$ at a given kinetic energy $E_{k}^{m}$. In other words, a kinetic energy distribution measurement is made at the matching ionic charge $q=q_{m}$ and a ionic charge distribution measurement is made at the matching kinetic energy $E_{k}=E_{k}^{m}$. The underlying hypothesis is that the kinetic energy distribution does not depend on the ionic charge (and respectively) which is not true as demonstrated in Fig. 2. Therefore a correction of the eq. (3) must be done in order to correctly estimate the relative mass yield $\mathcal{N}(A)$. Concretely, average and width of the kinetic energy distributions are extracted as function of the ionic charge. A linear fit is then performed:

$$
\begin{aligned}
\overline{E_{k}}(q) & =a q+b \\
\sigma_{E_{k}}(q) & =c q+d
\end{aligned}
$$

Then the correction due to the $\left(E_{k}, q\right)$ correlation is performed through the ionic charge distribution. This correction is made in order to answer to the following question: what would be the weight of each $P\left(q, E_{k}^{m}\right)$ if the measurement was made at a different kinetic energy? $P\left(q_{m}, E_{k}^{m}\right)$ is corrected through:

$$
P^{\text {corr }}\left(q_{m}, E_{k}^{m}\right)=\frac{P\left(q_{m}, E_{k}^{m}\right) \exp \left(-\frac{\left(\overline{E_{k}}\left(q_{m}\right)-E_{k}^{m}\right)^{2}}{\sigma_{E_{k}}\left(q_{m}\right)}\right)}{\sum_{q} P\left(q, E_{k}^{m}\right) \exp \left(-\frac{\left(\overline{E_{k}}(q)-E_{k}^{m}\right)^{2}}{\sigma_{E_{k}}(q)}\right)}
$$

In this expression, the kinetic energy distribution is assumed Gaussian, which is a good approximation when using a thin target (typically under $100 \mu \mathrm{g} . \mathrm{cm}^{-2}$ ). With this new procedure, a relative mass yield can be estimated for different ionic charges $q$ :

$$
\mathcal{N}(A \mid q)=\frac{\sum_{E_{k}} \mathcal{N}\left(A, q, E_{k}\right)}{P^{c o r r}\left(q, E_{k}^{m}\right)}
$$

The mean value is derived using the following formula:

$$
\overline{\mathcal{N}}(A)=\left(\sum_{i, j}^{n, n}\left(C^{-1}\right)_{i, j}\right)^{-1}\left(\sum_{i, j}^{n, n}\left(C^{-1}\right)_{i, j} \mathcal{N}\left(A \mid q_{i}\right)\right)
$$

with $C$ the covariance matrix between each $\mathcal{N}\left(A, q_{i}\right)$ and $n$ the number of kinetic energy distributions. The compatibility between each $\mathcal{N}\left(A, q_{i}\right)$ is achieved through a generalized $\chi^{2}$ test with a confidence level of $90 \%$. If the test is not passed, an uncertainty is incrementally added on the diagonal value of $C$ [12]. This additional uncertainty can be interpreted as the limits of the approximations made (linear fit used in eq. (4)) and as the consequence of the LOHENGRIN stability. Figure 3 shows the impact of the correlation $\left(E_{k}, q\right)$. Both the uncertainty and the bias are reduced. In order to produce independent 

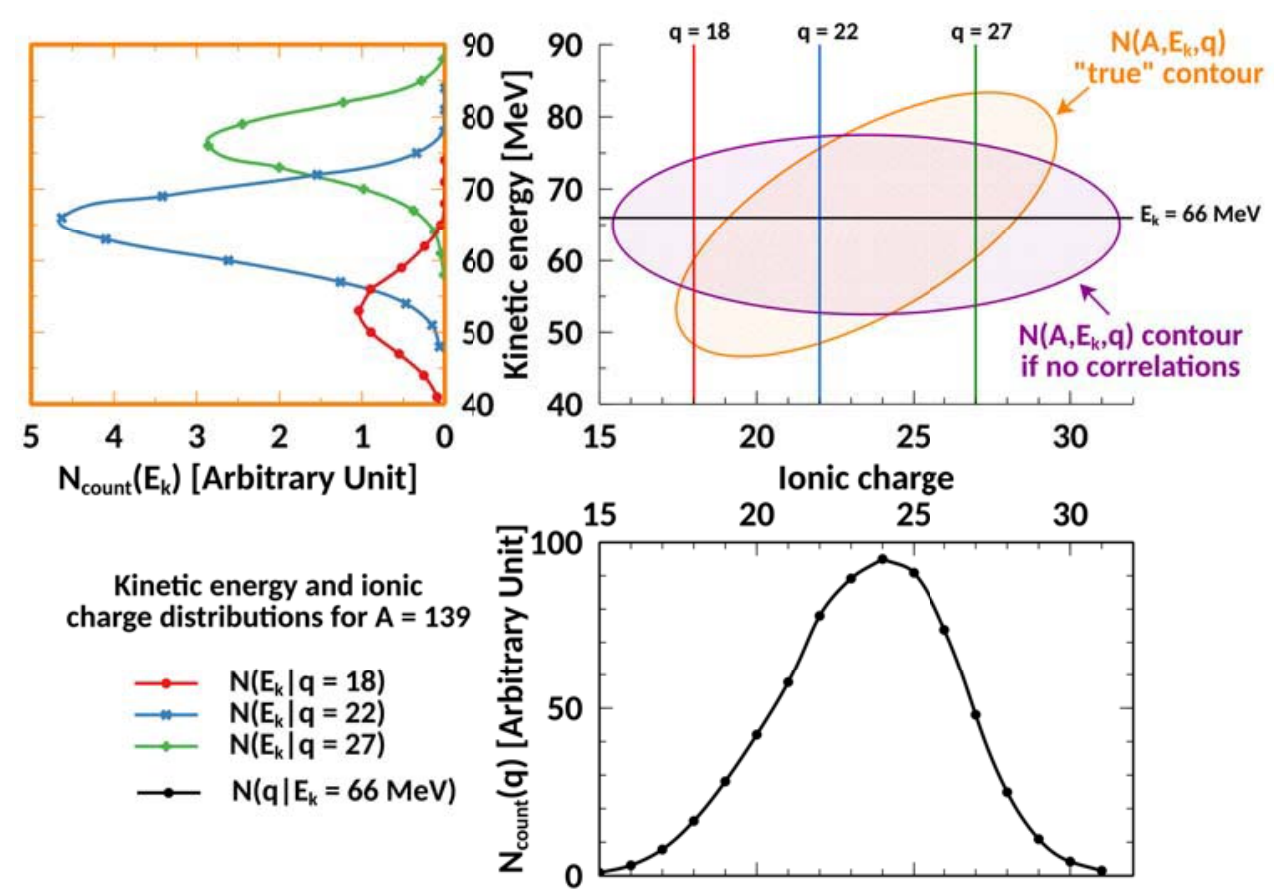

Figure 2. Dependence of the kinetic energy distribution as function of the ionic charge. The $\mathcal{N}\left(A, q, E_{k}\right)$ contour is then rotated thanks to the correlation between $\left(E_{k}, q\right)$. Data are coming from the study on the ${ }^{241} \mathrm{Pu}\left(n_{t h}, f\right)$ reaction.

data, the whole heavy (or light peak) must be measured (at least $99.5 \%$ ). Then the absolute mass yields $Y(A)$ are written:

$$
Y(A)=\frac{\mathcal{N}(A)}{\sum_{A \in \text { Heavy }} \mathcal{N}(A)}
$$

With this procedure the final uncertainty is of the order of $3 \%$ for the highly produced masses $(Y(A)>$ $1 \%)$. The covariance matrix can also be derived reflecting the raw-data analysis process.

\section{Measurements of fission product isotopic yields $Y(A, Z)$}

To assess the nuclear charge of the fission products (in the heavy mass region), $\gamma$ spectroscopy is used on the LOHENGRIN recoil spectrometer. A movable tape is placed inside a vacuum chamber surrounded by two High Purity Germanium (HPGe) clovers (with 4 Ge crystals each). The setup is positioned at the focal position 2. The procedure for measuring isotopic yields is described hereafter. First kinetic energy distributions and ionic charge distribution are measured with the IC (placed at the focal position 1). Then the ionic charge distribution is also measured with the HPGe and allows to determine $N(Z \mid A)$ which corresponds to the relative isotopic yields. Finally the isotopic yields is 


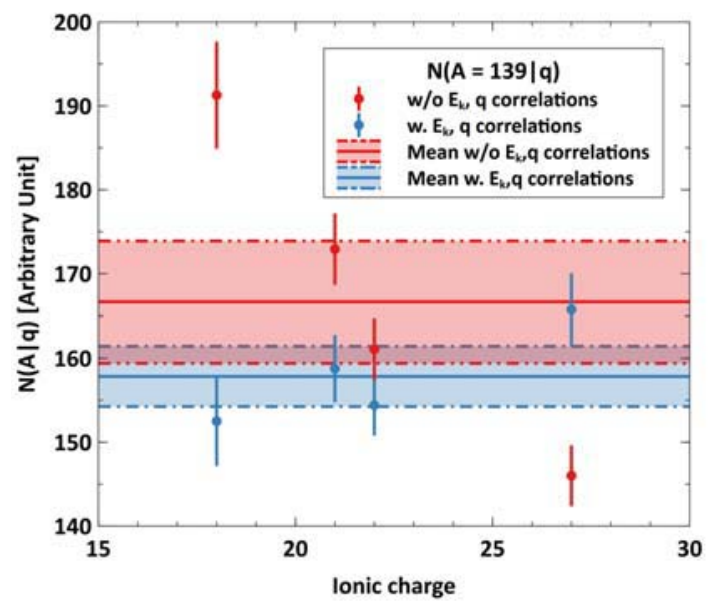

Figure 3. Estimation of the relative mass yield for $A=139$ from the ${ }^{241} \mathrm{Pu}\left(n_{t h}, f\right)$ reaction. Taking into account the correlation $\left(E_{k}, q\right)$ permits to reduce the uncertainty and the bias introduced by the classical procedure to estimate the fission mass yields on LOHENGRIN.

given by:

$$
\begin{aligned}
Y(A, Z) & =N(Z \mid A) \times k_{139} \\
k_{139} & =Y(A=139) / \sum_{Z} N(Z \mid A=139)
\end{aligned}
$$

In this method, the mass $A=139$ is supposed to be completely measured through $\gamma$ spectroscopy. Moreover, some corrections [5, 13] are needeed to transform the $\gamma$ count rate $N_{\gamma}\left(Z, q, E_{k}^{m}\right)$ to the relative isotopic yield $\mathrm{N}(\mathrm{Z} \mid \mathrm{A})$.

Here a focus on the impact of $\gamma$ intensity $I_{\gamma}$ is made. Indeed in the case of the ${ }^{136} \mathrm{Te}$, two different sets of $\gamma$ rays are in agreement. As shown on Fig. 4 (left), to assess the production rate of ${ }^{136} \mathrm{I}$, coming from fission, the $\beta^{-}$decay of ${ }^{136} \mathrm{Te}$ to ${ }^{136} \mathrm{I}$ must be corrected. A negative solution is found for the solution 2 as shown on Fig. 4 (right). In other words, the relative intensity between those $\gamma$ are not consistent. This example shows how the isotopic yields are sensitive to the nuclear structure parameters.

\section{Other observables of interest to study the fission process}

Thanks to the versatility of the LOHENGRIN recoil spectrometer, indirect fission observables can be studied. For instance using the mass yield measurements, the mean kinetic energy as function of mass can be derived [14]. This quantity can be compared with other measurements and can be used by different models such as FIFRELIN $[15,16]$ to validate the input data and the underlying models. The study of the symmetry region can give insight on the Brosa fission modes [17] by looking at the kinetic energy distributions [18]. The local odd-even effect [13] give some information on the pairs breaking but also on the neutron emission process. An experimental program on isomeric ratios (IRs) measurements was developed since 2012 [19]. Especially a focus on the evolution of IR as function 

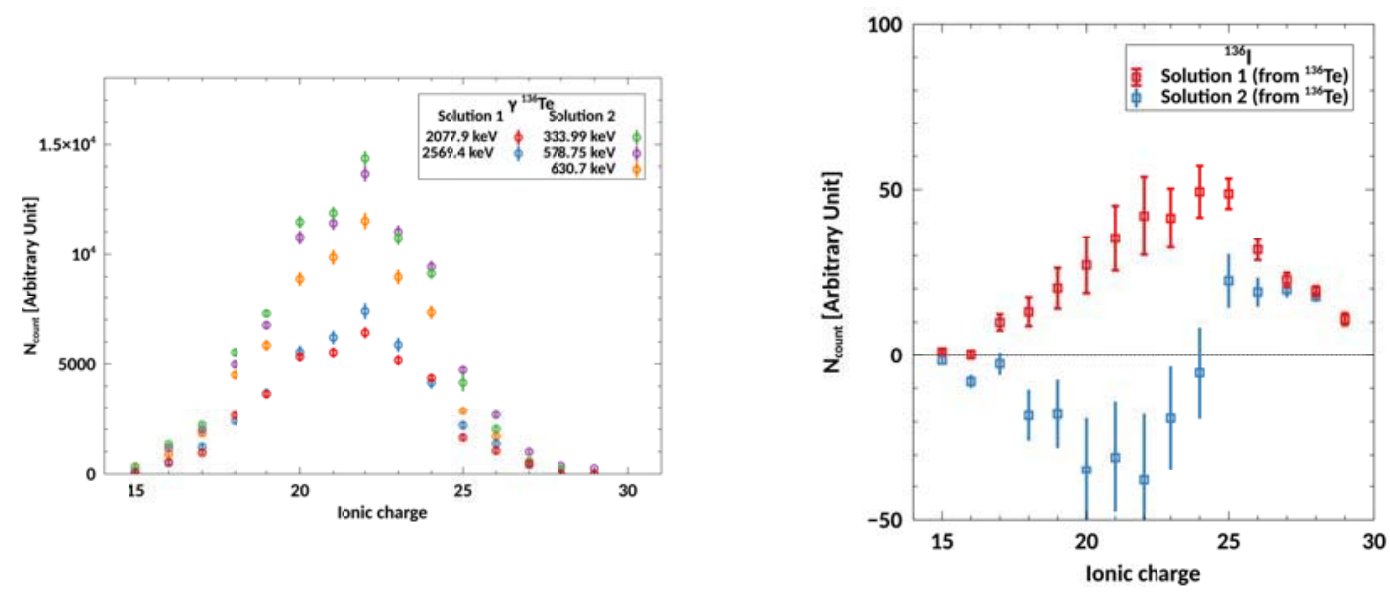

Figure 4. Count rate extracted from $\gamma$ spectrum (left). Tow different sets of $\gamma$-rays (solution 1 and solution 2) are in agreement within each other. Correction from $\beta^{-}$decay of ${ }^{136} \mathrm{Te}$ to ${ }^{136}$ I give rise to two contradictory solutions (right). Data are coming from the studies of the ${ }^{241} \mathrm{Pu}\left(n_{t h}, f\right)$ reaction.

of fission product kinetic energy was made [20, 21]. Using de-excitation code such as FIFRELIN, an angular momentum distribution can be derived. These data can also be used to test the level density or $\gamma$-strength function models as well as the coherency of the nuclear level schemes. Finally looking at the structures in the kinetic energy distributions coming from a high quality thin target allows to make an indirect measurement of the neutron emission process for a given mass.

\section{Conclusion}

This work presents the new procedure developed on the LOHENGRIN recoil spectrometer in order to measure mass and isotopic yields. Taking into account the correlation $\left(E_{k}, q\right)$ permits to improve the accuracy and uncertainties of the measurements. The strong sensibility of isotopic yield on nuclear structure parameters was highlighted. Thanks to the performance of the spectrometer, indirect measurement of observable of interest are currently studied in order to test fission models or nuclear structure models.

\section{Acknowledgment}

This work was supported by IN2P3, by the University of Grenoble Alpes and by "le défi NEEDS". The authors are grateful for the support of the ILL and all the staff involved from CEA-Cadarache and LPSC.

\section{References}

[1] V. Vallet et al., Proc. Physor 2014, 2014-003 (2015)

[2] A. Tsilanizara et al., Ann. Nucl. Energy 90, 62-70 (2016)

[3] M. Tiphine et al., Proc. Global 2015, 5327 (2015)

[4] P. Armbruster et al., Nucl. Instrum. Methods 139, 213 (1976) 
[5] A. Bail et al., Phys. Rev. C, 84, 034605 (2011)

[6] Y.K. Gupta et al., Phys. Rev. C, 96, 014608 (2017)

[7] C. Amouroux et al., EPJ Web Conf. 62, 06002 (2013)

[8] O. Serot et al., Nucl. Data Sheets, 119, 320-323 (2014)

[9] A. Chebboubi et al., EPJ Web Conf. 146, 04021 (2017)

[10] S. Julien-Lafferière et al., ND2019 Conference, to be published

[11] G. Fioni et al., Nucl. Instrum. Meth. A 332, 175 (1993)

[12] S. Julien-Lafferière et al., EPJ Nucl. Sci. Technol. 4, 25 (2018)

[13] S. Julien-Lafferière et al., to be submitted

[14] S. Julien-Lafferière et al., EPJ Web Conf. 169, 00008 (2018)

[15] O. Litaize and O. Serot, Phys. Rev. C 82, 054616 (2010)

[16] O. Litaize et al., EPJ A 51, 1 (2015)

[17] U. Brosa et al., Phys. Rep. 197, (4) 167-262 (1990)

[18] A. Chebboubi et al., EPJ Web Conf. 111, 08003 (2016)

[19] A. Chebboubi et al., EPJ Web Conf. 111, 08002 (2016)

[20] A. Chebboubi et al., Phys. Lett. B 775, 190-195 (2017)

[21] J. Nicholson et al., Theory-5 Conference, to be published 